\title{
Ergonomics study for workers at food production industry
}

\author{
Hamizatun Mohd Fazi ${ }^{1, *}$, Nik Mohd Zuki Nik Mohamed ${ }^{1}$, Mohd Fadzil Faisae Ab \\ Rashid $^{1}$ and Ahmed Nasser Mohd Rose ${ }^{1}$ \\ ${ }^{1}$ Faculty Mechanical Engineering, University Malaysia Pahang, 26600 Pekan, Pahang
}

\begin{abstract}
The health constraint faced by production workers affects the quality of the work. The productivity of the workers is affected by the Work-related Musculoskeletal Disorder (WMSD) which limits the movement of the workers. The comfort workplace condition, known as ergonomic environment is important to prevent the occurrence of the WMSD. Proper ergonomic workplace considers the condition of the workers while doing the assigned work. The objectives of this study are to identify the current problems related to ergonomic in food production process, to analyse the actual production data by using Rapid Upper Limb Assessment (RULA) and Rapid Entire Body Assessment (REBA) and to recommend the ergonomic workplace environment based on the condition of the study. The study was done at a Small and Medium Enterprises (SME) food production company in the Klang Valley of Malaysia. The condition of the workers affects the productivity of the company due to workers' health deficiency. From the findings, the workers are exposed to the awkward postures which leads to the Work-Musculoskeletal Disorders (WMSDs). Besides, the best height of the worker at the study area (critical area) to prevent the worker from WMSDs is within $155 \mathrm{~cm}$ to $160 \mathrm{~cm}$. The results show that the workers are exposed to the WMSD in different level of risks which causes high absenteeism among the workers.
\end{abstract}

\section{Introduction}

As a developing country, Malaysia faced a rapid industrial growth in various sectors such as foods and services sector. The contributions of every sector are really important to generate a stable economy towards the glorious nation. The food industries in Malaysia had vast growth and contributed to the economy improvement of the country as food is necessity for the human beings [1]. In the food manufacturing sector, the health of the employee may be exposed to the hazards such as the work-related musculoskeletal disorder. Normally, the injuries happen either at the muscles, tendons, nerves, blood vessel or ligaments.

Musculoskeletal disorder (MSDs) may occur because of continuously performing repetitive tasks, working in repeated and sustained or difficult postures, performing strenuous physical work, and using forceful exertion [2]. To improve the productions for

\footnotetext{
*Corresponding author: hamizatunfazi@gmail.com
} 
higher profit, employee productivity is important [3]. Work productivity as an indicator has been a general subject for examination in several studies on musculoskeletal disorders that influence the workers condition [4]. If the workers facing health problem, it indicates that the losses faced by the company [5]. Heavy lifting and awkward work postures are the physical working condition that relate with sick-leave [6]. As the condition of the workers affects the productivity, hence the worker needs to have a comfortable workplace which is ideally free from hazards.

The comfortable workplace is known as the proper ergonomic working environment. Ergonomics is the information such as the behavior of the human, limitation and capacity of human that applies on the machines, designs of tools, tasks, and environment for secure, comfort and beneficial for human use [7]. A bad worksite design leads to the difficulties for the workers such as fatigue and injuries. Besides, the injuries relate to the low productivity of the workers and increase the cost to the company where the workers need to rest and the company needs to bear the losses [8]. In the study of the occupational risks, there are few ergonomics analysis tools available to determine the risks of the worker at the workplace. There methods identified and classified the risks into several parts which are self-reports, observational methods and direct measurements [9]. In this study, the ergonomic tool used to identify the occupational risks on the workers are Rapid Upper Limb Assessment (RULA) and Rapid Entire Body Assessment (REBA). The selection of the RULA and REBA are based on the tasks doing by the workers which are awkward, repetitive work and involves the entire body parts.

\section{Methodology}

This study was conducted at one of the food industry in Malaysia. The first step for this research was to study the process flow of the food processing by assessing the overall working areas. Then, by doing interview sessions and observations, the critical workplace was identified. The chosen subjects were from the filling and stamping workstation since it was the critical workplace. The subjects are taken from both gender while the height of the workers are within $150 \mathrm{~cm}$ to $180 \mathrm{~cm}$. Next, the subjects were required to conduct their working cycle as usual and the pictures and videos of the process were taken. The postures that were repetitive and awkward were chosen. Later, the RULA and REBA analysis were performed to assess the subjects' posture level of discomfort. Figure 1 and Figure 2 show the RULA and REBA assessments method used in this study. The level of MSD risk for RULA is shown in Table 1 while the level of MSD risk for REBA is shown in Table 2.

The movements of the workers were divided into two different positions which were Posture 1 and Posture 2. Posture 1 was the positions where the workers were taking the products from the filling and the stamping machine and put the products into the basin filling water to prevent the products from defective. The position where the workers were arranging the products in the container is called Posture 2.

The movements of the workers were divided into two different positions which were Posture 1 and Posture 2. Posture 1 was the positions where the workers were taking the products from the filling and the stamping machine and put the products into the basin filling water to prevent the products from defective. The position where the workers were arranging the products in the container is called Posture 2. 


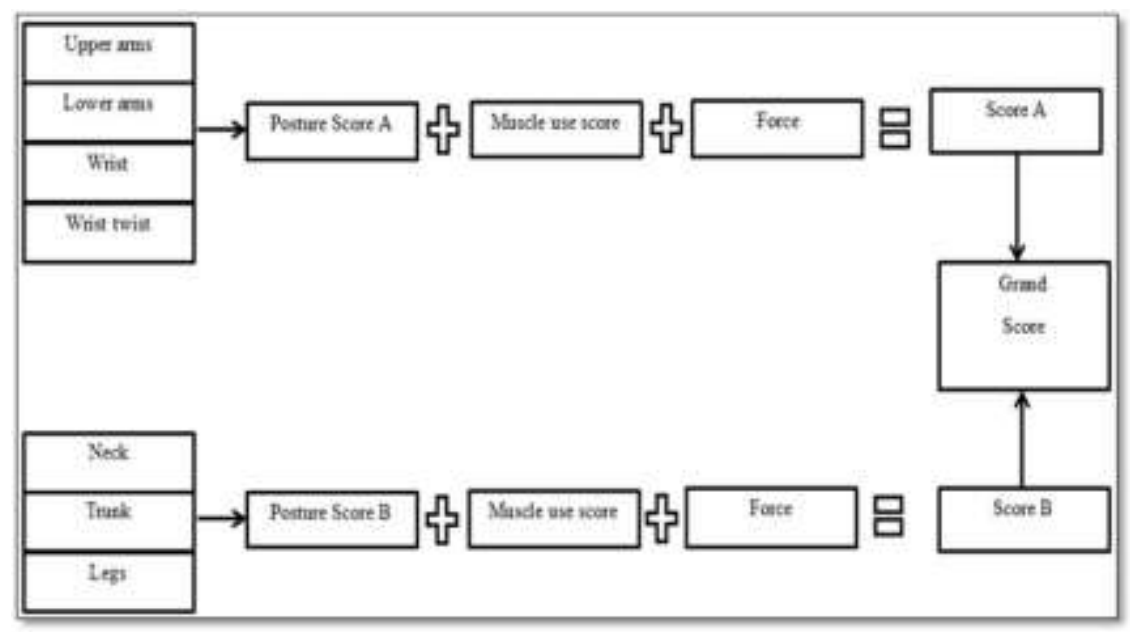

Fig. 1. RULA assessment method.



Fig. 2. REBA assessment method.

Table 1. Level of MSD Risk (RULA) [10].

\begin{tabular}{|c|c|}
\hline Score & Level of MSD risk \\
\hline $1-2$ & Negligible risk, no action required \\
\hline $3-4$ & Low risk, change may be need \\
\hline $5-6$ & Medium risk, further investigation, change soon \\
\hline $6+$ & Very high risk, implement change now \\
\hline
\end{tabular}

Table 2. Level of MSD Risk (REBA) [11].

\begin{tabular}{|c|c|}
\hline Score & Level of MSD risk \\
\hline 1 & Negligible risk, no action required \\
\hline $2-3$ & Low risk, change may be need \\
\hline $4-7$ & Medium risk, further investigation, change soon \\
\hline $8-10$ & Very high risk, implement change now \\
\hline $11+$ & Very high risk, implement change \\
\hline
\end{tabular}




\section{Results and discussion}

From the interviews and the observations, it was shown clearly that the subjects were exposed to the awkward postures during long working hours. There were two postures chosen involving three subjects for each posture. From the subjects working cycle recorded, the assessments were carried out to identify the problems. Figure 3 shows the RULA grand scores versus postures for all three workers that involved in this study. The results were analysed by referring to the level of MSD risk for RULA in Table 1.

The result for the RULA grand score is shown in Figure 3. For Posture 1, it shows that worker $\mathrm{A}$ and $\mathrm{C}$ are exposed in the high risks that need to be changed immediately since both scores are 7 while worker B is in medium risks that need further investigations as the score is 6 . Based on the results, it indicates that the workplace is too hazardous for workers A and C. For Posture 2, the graph denotes that worker A is highly exposed to MSD as the score is 7. Worker B is still in the low-risk condition while worker $\mathrm{C}$ is in medium risk condition. The score for workers $\mathrm{B}$ and $\mathrm{C}$ are 4 and 6 respectively. The results for RULA assessment reveal that worker $\mathrm{A}$ is in the high risks for both postures. The change is needed immediately to prevent the worker A from MSD. Besides, by comparing the results for each posture, all workers are in awkward condition at Posture 1. Therefore, the condition of the workplace for Posture 1 should be improved.



Fig. 3. RULA grand scores versus postures.

The graphical results for REBA scores versus postures are visualized in Figure 4. The results are analyze by referring to the level of MSD risk for REBA as in Table 2. From the graph in Figure 4, it is found that workers B and C are exposed in the medium risks that need implement change in the near future, since both scores are 6 while worker A is in very high risks as the score is 9. The Posture 1 is really dangerous to worker A and needs the implementation of the changes immediately. 


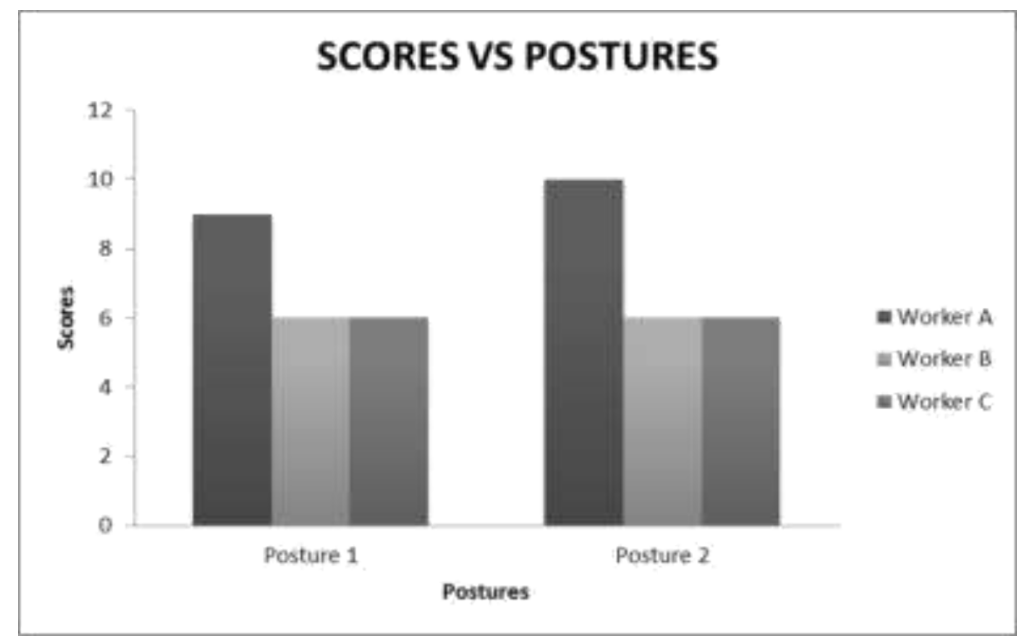

Fig. 4. REBA grand scores versus postures.

Based on the results for Posture 2, it indicates that the position is really at high risk level for worker A as its score is 10. Furthermore, the graph denotes that workers B and C are in medium risk condition. Both scores for worker $\mathrm{B}$ and $\mathrm{C}$ are 6 . The results for REBA assessment divulge that worker $\mathrm{A}$ is in the high risks for both postures. The change is needed to be implemented immediately to ensure that the workers are free from MSD.

The comparison between the RULA and REBA are made in Figure 5. The graph indicates that the worker $\mathrm{A}$ is always in the awkward condition as compared to the other workers as the grand scores for A are always higher than 6 which represent the medium to high risks states. Worker $\mathrm{A}$ is the tallest participants among the others. The results reveal that the worker who has the height of $172 \mathrm{~cm}$ is not suitable to work at the study area. Therefore, worker A should be transferred to the other workstations.

Contrarily, the highest scores for worker B and C are 6 and 7 respectively which are still in the medium risk. Therefore, conclusion can be made from the outcomes of the study where the heights of the workers affect the comfortable condition in the workplace. These studies reveal that the height of the worker which suitable to do the job task at the study area and both posture is in the range of $155 \mathrm{~cm}-160 \mathrm{~cm}$ only.

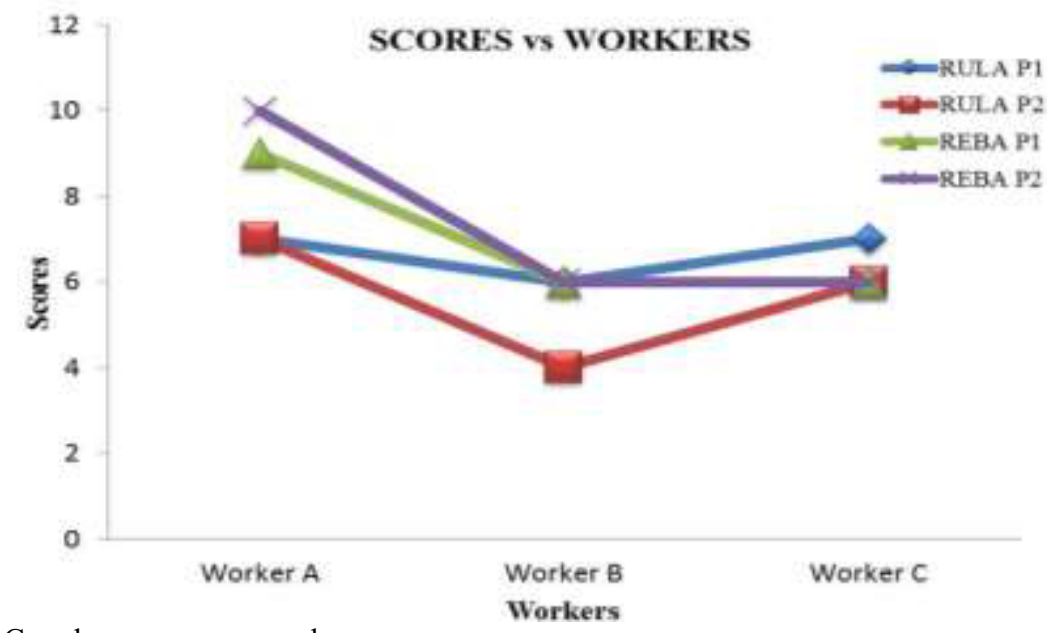

Fig. 5. Grand scores versus workers. 


\section{Recommendations}

Based on the study, below are the items that need to be implemented:

- Worker's Hiring Standard - The suitable height that resulted from the research should be within the range of $155 \mathrm{~cm}-160 \mathrm{~cm}$, therefore, it can be set as the standard or the compulsory requirement for the company to hire new workers. The anthropometry data of the workers are important to prevent the WMSDs that lead to the absenteeism and poor condition of the workers.

- Conveyor - The conveyor to connect the filling and stamping machine process. The height of the conveyor should be able to be adjusted to the ordinary height of the workers within the range of $75 \mathrm{~cm}-80 \mathrm{~cm}$ based on the samples study. The improved layout helps the workers from the MSD problem where the workers just sit while ensuring the product moves smoothly on the conveyor. Besides, the job task of the workers also decreases since the products directly move to the expiry stamping without the need to be moved manually.

- Using Ergonomic chairs - For this study, the ergonomics chairs are important to prevent the back pain and the lower part of the body while doing the assigned work. The elements of a good ergonomic chair that should be considered during selecting the chair which has lumbar support, foot rest and appropriate height level [12].

\section{Conclusions}

This paper has presented the approach to find out the occupational risk of the workers at the SME's food production company by using two types of analysis tools. The analysis tools used are RULA and REBA which are able to determine the condition of the workers at the critical areas which are not ergonomically designed. From the findings, the workers are exposed to the awkward posture which leads to the Work-Musculoskeletal Disorders (WMSDs). Besides, the best height of the worker at the study area (critical area) is within $155 \mathrm{~cm}$ to $160 \mathrm{~cm}$ to prevent the worker from WMSDs. Therefore, the employer should consider the height of the worker while placing the worker at the workstation.

\section{References}

1. Dolage, D.A.R., A.B. Sade, E.M. Ahmed, The influence of Flexible Manufacturing Technology adoption on productivity of Malaysian manufacturing industry. Economic Modelling, 2010. 27(1): p. 395-403.

2. Deros, B.M., D.D.I. Daruis, I.M. Basir, A Study on Ergonomic Awareness among Workers Performing Manual Material Handling Activities. Procedia - Social and Behavioral Sciences, 2015. 195: p. 1666-1673.

3. Dollard, M.F., D.Y. Neser, Worker health is good for the economy: Union density and psychosocial safety climate as determinants of country differences in worker health and productivity in 31 European countries. Social Science \& Medicine, 2013. 92: p. 114-123.

4. Andersen, L.N., et al., Efficacy of 'Tailored Physical Activity' on reducing sickness absence among health care workers: A 3-months randomised controlled trial. Manual Therapy, 2015. 20(5): p. 666-671.

5. Carrillo-Castrillo, J.A., et al., Estimation of the relative risks of musculoskeletal injuries in the Andalusian manufacturing sector. International Journal of Industrial Ergonomics, 2016. 52: p. 69-77.

6. Laaksonen, M., et al., Work Arrangements, Physical Working Conditions, and Psychosocial Working Conditions as Risk Factors for Sickness Absence: Bayesian 
Analysis of Prospective Data. Annals of Epidemiology, 2010. 20(5): p. 332-338.

7. Probst, C.A., et al., Human factors engineering approaches to patient identification armband design. Applied Ergonomics, 2016. 52: p. 1-7.

8. Falck, A.-C., M. Rosenqvist, A model for calculation of the costs of poor assembly ergonomics (part 1). International Journal of Industrial Ergonomics, 2014. 44(1): p. 140-147.

9. Kathy Cheng, H.-Y., C.-Y. Cheng, Y.-Y. Ju, Work-related musculoskeletal disorders and ergonomic risk factors in early intervention educators. Applied Ergonomics, 2013. 44(1): p. 134-141.

10. McAtamney, L., E. Nigel Corlett, RULA: a survey method for the investigation of work-related upper limb disorders. Applied Ergonomics, 1993. 24(2): p. 91-99.

11. Hignett, S., L. McAtamney, Rapid Entire Body Assessment (REBA). Applied Ergonomics, 2000. 31(2): p. 201-205.

12. Amick Iii, B.C., et al., A field intervention examining the impact of an office ergonomics training and a highly adjustable chair on visual symptoms in a public sector organization. Applied Ergonomics, 2012. 43(3): p. 625-631. 